\title{
Appendix B: Forecast detail
}

Table BI. Real GDP growth and inflation

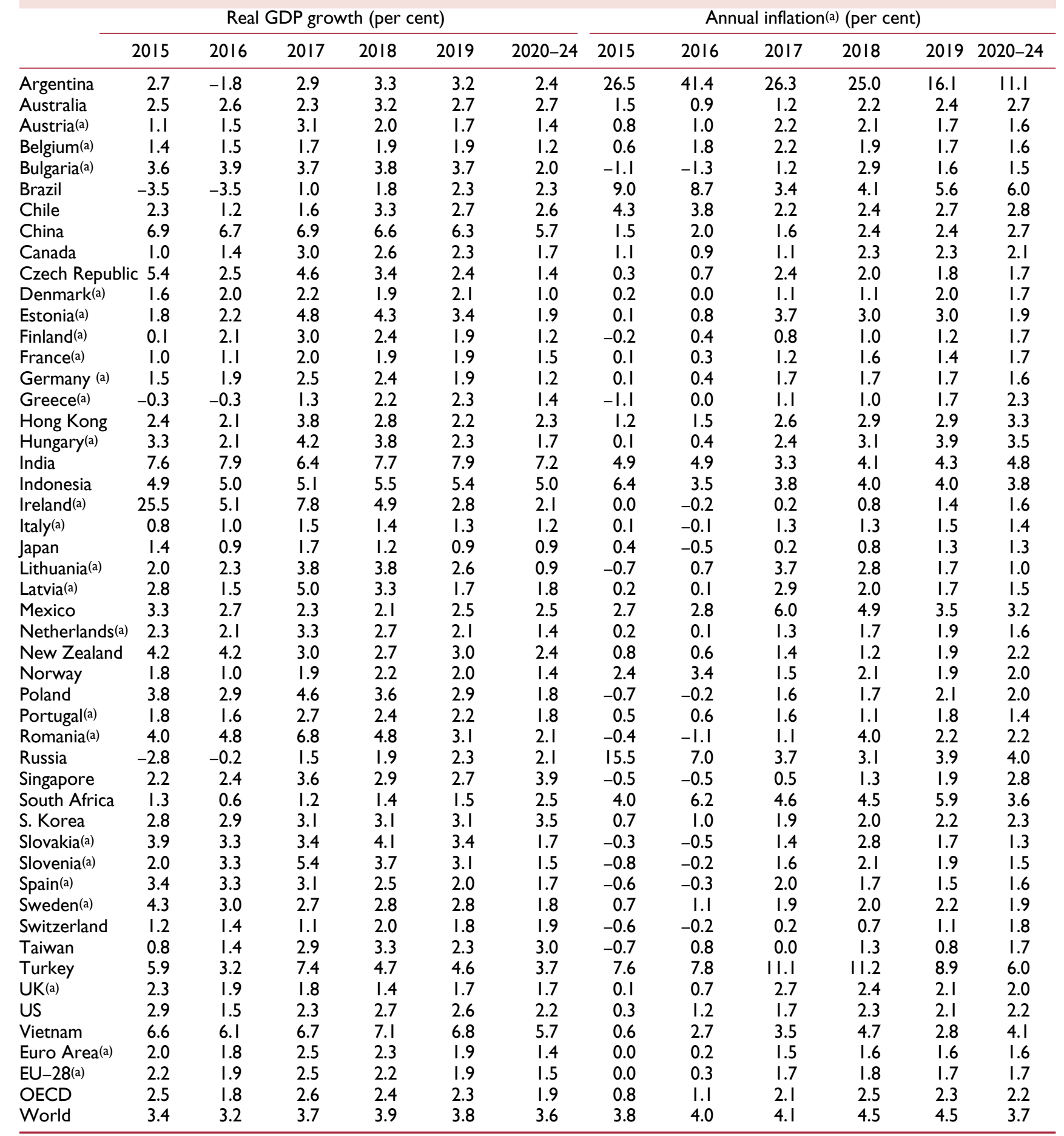


Table B2. Fiscal balance and government debt

Fiscal balance (per cent of GDP)(a)

Government debt (per cent of GDP, end year)(b)

\begin{tabular}{|c|c|c|c|c|c|c|c|c|c|c|c|c|}
\hline & 2015 & 2016 & 2017 & 2018 & 2019 & 2024 & 2015 & 2016 & 2017 & 2018 & 2019 & 2024 \\
\hline Australia & -1.5 & -2.1 & -1.7 & -1.6 & -1.0 & -1.2 & 43.9 & 44.9 & 45.5 & 45.2 & 44.1 & 38.2 \\
\hline Austria & -1.0 & -1.6 & -0.5 & -0.6 & -0.5 & -1.3 & 84.3 & 83.5 & 80.3 & 77.9 & 74.9 & 67.4 \\
\hline Belgium & -2.5 & -2.5 & -1.8 & -1.1 & -0.7 & -2.4 & 106.0 & 105.7 & 103.9 & 100.0 & 96.7 & 92.3 \\
\hline Bulgaria & -1.6 & 0.0 & 0.3 & 0.3 & 0.1 & -0.7 & - & - & - & - & - & - \\
\hline Canada & -0.1 & -1.1 & -1.0 & -1.0 & -1.0 & -1.5 & 97.8 & 96.6 & 95.6 & 91.6 & 88.2 & 79.6 \\
\hline Czech Rep. & -0.6 & 0.7 & 0.9 & 0.4 & 0.0 & -1.4 & 38.7 & 35.8 & 33.4 & 31.5 & 30.5 & 31.1 \\
\hline Denmark & -1.8 & -0.6 & -2.4 & -1.5 & -1.3 & -1.4 & 39.5 & 37.7 & 36.4 & 36.8 & 36.8 & 39.0 \\
\hline Estonia & 0.1 & -0.3 & -0.6 & -0.7 & -0.8 & -1.3 & - & - & - & - & - & - \\
\hline Finland & -2.7 & -1.7 & 0.0 & 0.6 & 0.2 & -1.6 & 63.6 & 63.1 & 61.0 & 58.5 & 56.6 & 53.8 \\
\hline France & -3.6 & -3.4 & -2.6 & -1.9 & -1.7 & -2.7 & 95.8 & 96.6 & 96.7 & 95.2 & 93.7 & 88.3 \\
\hline Germany & 0.6 & 0.8 & I.I & 0.9 & 0.5 & -1.2 & 71.0 & 68.2 & 64.1 & 59.6 & 56.2 & 47.1 \\
\hline Greece & -5.7 & 0.4 & -1.2 & -1.3 & -0.3 & -0.1 & 176.8 & 180.8 & 179.5 & 176.3 & 165.5 & 132.5 \\
\hline Hungary & -2.0 & -1.9 & -3.0 & -3.0 & -2.8 & -2.4 & 74.4 & 73.7 & 68.8 & 67.7 & 66.4 & 61.9 \\
\hline Ireland & -1.9 & -0.7 & 0.8 & 0.6 & -0.2 & -1.2 & 77.0 & 72.9 & 70.2 & 67.3 & 64.6 & 58.6 \\
\hline Italy & -2.6 & -2.5 & -1.6 & -1.1 & -1.0 & -2.4 & 131.6 & 131.9 & 132.1 & 128.2 & 124.7 & 116.0 \\
\hline Japan & -3.5 & -4.6 & -4.8 & -4.2 & -4.0 & -4.2 & 213.9 & 216.8 & 218.2 & 220.9 & 219.6 & 211.0 \\
\hline Lithuania & -0.2 & 0.3 & 0.4 & 0.1 & -0.2 & -1.2 & - & - & - & - & - & - \\
\hline Latvia & -1.2 & 0.0 & 0.2 & 0.1 & -0.1 & -0.8 & - & - & - & - & - & - \\
\hline Netherlands & -2.1 & 0.4 & 1.3 & 2.0 & 1.7 & -1.2 & 64.6 & 61.8 & 55.9 & 51.9 & 48.2 & 41.8 \\
\hline Poland & -2.6 & -2.5 & -2.0 & -1.7 & -1.6 & -2.2 & 51.7 & 53.8 & 50.5 & 49.6 & 49.0 & 49.9 \\
\hline Portugal & -4.4 & -2.0 & -1.6 & -1.4 & -1.1 & -1.7 & 128.8 & 130.1 & 127.4 & 124.9 & 121.8 & 111.0 \\
\hline Romania & -0.8 & -3.0 & -3.1 & -3.1 & -2.9 & -1.9 & - & - & - & - & - & - \\
\hline Slovakia & -2.7 & -2.2 & -1.6 & -1.0 & -0.6 & -0.2 & - & - & - & - & - & - \\
\hline Slovenia & -2.9 & -1.9 & -1.0 & -1.0 & -1.2 & -1.8 & - & - & - & - & - & - \\
\hline Spain & -5.3 & -4.5 & -3.3 & -2.1 & -1.8 & -2.3 & 99.4 & 99.0 & 97.5 & 94.7 & 92.4 & 85.5 \\
\hline Sweden & 0.2 & I.I & 0.9 & 1.4 & 1.0 & -0.6 & 44.2 & 42.3 & 39.1 & 35.6 & 32.8 & 27.4 \\
\hline UK & -4.2 & -3.0 & -1.9 & -4.0 & -3.8 & -3.9 & 88.2 & 88.2 & 86.0 & 86.1 & 84.6 & 79.3 \\
\hline US & -4.3 & -5.0 & -3.6 & -5.4 & -5.3 & -3.2 & 104.1 & 105.3 & 103.2 & 103.4 & 104.0 & 100.9 \\
\hline
\end{tabular}

Notes: (a) General government financial balance; Maastricht definition for EU countries. (b) Maastricht definition for EU countries.

Figure $\mathrm{BI}$. World GDP is estimated to have expanded by 4 per cent (year-on-year) in the fourth quarter of 2017

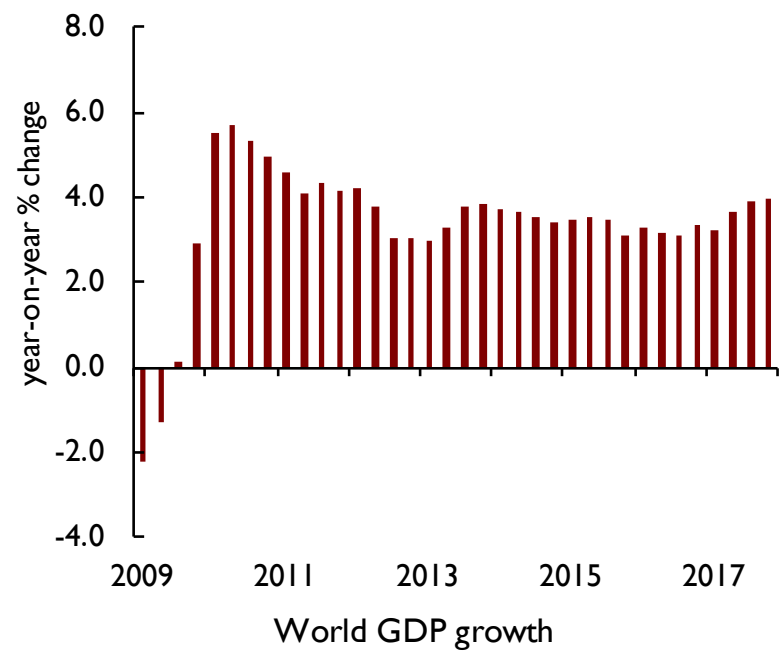

Figure B2. NIESR estimates that world trade grew by 4.2 per cent (year-on-year) in 2017Q4

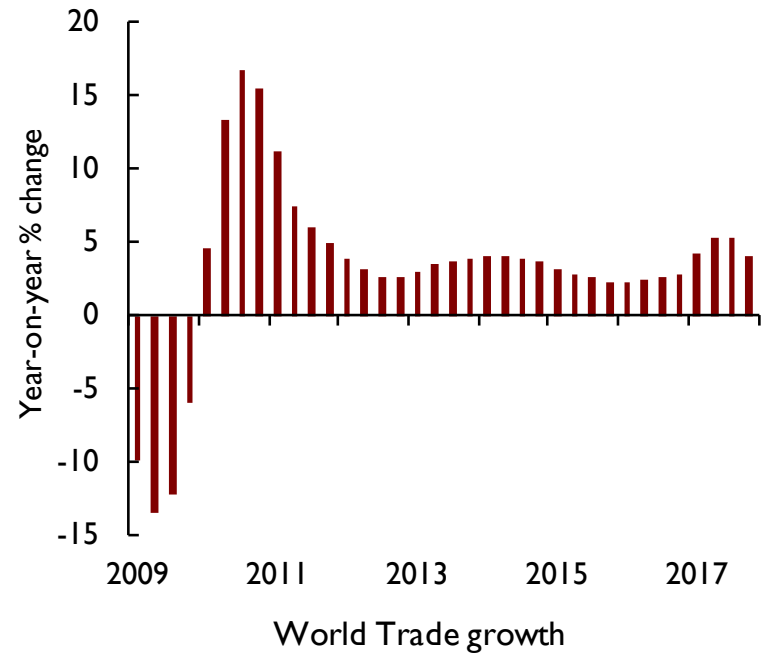


Table B3. Unemployment and current account balance

\begin{tabular}{|c|c|c|c|c|c|c|c|c|c|c|c|c|}
\hline & \multicolumn{6}{|c|}{ Standardised unemployment rate } & \multicolumn{6}{|c|}{ Current account balance (per cent of GDP) } \\
\hline & 2015 & 2016 & 2017 & 2018 & 2019 & $2020-24$ & 2015 & 2016 & 2017 & 2018 & 2019 & $2020-24$ \\
\hline Australia & 6.1 & 5.7 & 5.6 & 5.5 & 5.2 & 5.1 & -4.7 & -3.1 & -2.3 & -2.8 & -2.4 & -2.6 \\
\hline Austria & 5.7 & 6.0 & 5.5 & 5.2 & 5.2 & 4.6 & 1.9 & 2.1 & 2.8 & 1.0 & 1.2 & 1.1 \\
\hline Belgium & 8.5 & 7.8 & 7.1 & 6.4 & 6.7 & 6.2 & -0.2 & 0.1 & -0.7 & -1.6 & -1.4 & 0.1 \\
\hline Bulgaria & 9.1 & 7.5 & 6.2 & 5.2 & 5.3 & 5.9 & 0.0 & 2.3 & 4.6 & 4.4 & 5.4 & 2.9 \\
\hline Canada & 6.9 & 7.0 & 6.3 & 5.8 & 5.8 & 6.0 & -3.6 & -3.2 & -3.0 & -2.5 & -1.8 & -0.9 \\
\hline China & - & - & - & - & - & - & 2.8 & 1.8 & I.3 & 0.5 & 0.5 & 0.9 \\
\hline Czech Rep. & 5.0 & 3.9 & 2.9 & 2.1 & 1.9 & 2.6 & 0.2 & 1.5 & 0.9 & -0.5 & -1.1 & -1.3 \\
\hline Denmark & 6.2 & 6.2 & 5.7 & 4.7 & 4.7 & 4.8 & 8.8 & 7.3 & 7.7 & 6.9 & 7.2 & 8.3 \\
\hline Estonia & 6.2 & 6.8 & 5.8 & 6.4 & 6.0 & 6.5 & 1.9 & 1.9 & 3.2 & 1.7 & I.I & 0.3 \\
\hline Finland & 9.3 & 8.9 & 8.6 & 8.4 & 8.4 & 8.3 & -0.7 & -0.4 & 0.7 & -0.2 & -0.1 & 0.7 \\
\hline France & 10.4 & 10.1 & 9.4 & 8.9 & 8.4 & 7.3 & -0.4 & -0.9 & -1.2 & -0.8 & -0.5 & -1.0 \\
\hline Germany & 4.7 & 4.2 & 3.8 & 3.6 & 3.6 & 4.0 & 9.0 & 8.5 & 8.1 & 7.9 & 7.5 & 7.4 \\
\hline Greece & 25.0 & 23.5 & 21.5 & 20.7 & 19.2 & 18.5 & -0.2 & -1.0 & -0.7 & -1.4 & -0.3 & -0.7 \\
\hline Hungary & 6.8 & 5.1 & 4.2 & 3.9 & 3.9 & 4.0 & 3.5 & 6.1 & 2.9 & 4.6 & 5.0 & 3.1 \\
\hline Ireland & 10.0 & 8.4 & 6.8 & 6.2 & 6.1 & 6.2 & 11.0 & 3.4 & 12.4 & 10.7 & 9.1 & 9.9 \\
\hline Italy & 11.9 & 11.7 & 11.3 & 10.8 & 10.5 & 10.5 & 1.5 & 2.7 & 3.0 & 3.1 & 3.9 & 4.9 \\
\hline Japan & 3.4 & 3.1 & 2.8 & 2.4 & 3.0 & 3.3 & 3.0 & 3.7 & 4.0 & 3.5 & 3.7 & 4.8 \\
\hline Lithuania & 9.1 & 7.9 & 7.1 & 7.4 & 7.7 & 7.8 & -2.4 & -1.1 & 1.0 & 0.1 & -2.4 & -3.3 \\
\hline Latvia & 9.9 & 9.6 & 8.7 & 8.1 & 7.5 & 6.6 & -0.5 & 1.4 & -0.8 & 0.7 & 0.5 & -0.7 \\
\hline Netherlands & 6.9 & 6.0 & 4.8 & 4.5 & 4.4 & 4.5 & 8.7 & 8.5 & 10.2 & 10.7 & 8.7 & 7.6 \\
\hline Poland & 7.5 & 6.2 & 4.9 & 4.4 & 4.5 & 4.2 & -0.6 & -0.3 & 0.2 & 0.8 & 1.0 & 0.1 \\
\hline Portugal & 12.6 & II.I & 9.0 & 7.8 & 7.9 & 8.0 & 0.3 & 0.6 & 0.6 & -0.4 & -1.4 & -1.4 \\
\hline Romania & 6.8 & 5.9 & 4.9 & 5.1 & 4.8 & 5.0 & -1.2 & -2.1 & -3.4 & -2.4 & -2.0 & -2.3 \\
\hline Slovakia & 11.5 & 9.7 & 8.1 & 7.3 & 7.4 & 8.2 & -1.7 & -1.5 & -2.1 & -0.9 & 0.2 & -0.6 \\
\hline Slovenia & 9.0 & 8.0 & 6.6 & 5.9 & 5.8 & 6.4 & 4.4 & 5.3 & 6.4 & 3.0 & 3.0 & 0.7 \\
\hline Spain & 22.1 & 19.6 & 17.2 & 15.0 & 13.2 & 13.1 & I.I & 1.9 & 2.0 & 1.7 & 2.3 & 2.1 \\
\hline Sweden & 7.4 & 6.9 & 6.7 & 6.1 & 6.2 & 6.7 & 4.5 & 4.2 & 3.2 & 3.9 & 4.4 & 5.5 \\
\hline UK & 5.4 & 4.9 & 4.4 & 4.1 & 4.2 & 4.7 & -5.2 & -5.8 & -4.1 & -4.0 & -3.8 & -3.5 \\
\hline US & 5.3 & 4.9 & 4.3 & 4.1 & 4.1 & 4.8 & -2.4 & -2.4 & -2.4 & -3.1 & -3.4 & -3.3 \\
\hline
\end{tabular}

Figure B3. China is not expected to become the world's biggest importer of goods and services before 2023

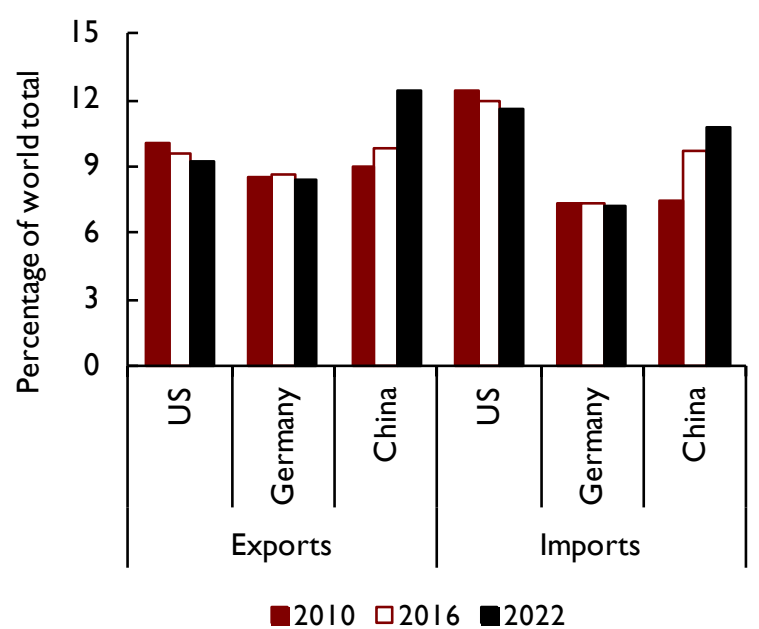

Figure B4. Since 2014, on a PPP basis, China has remained the world's largest economy

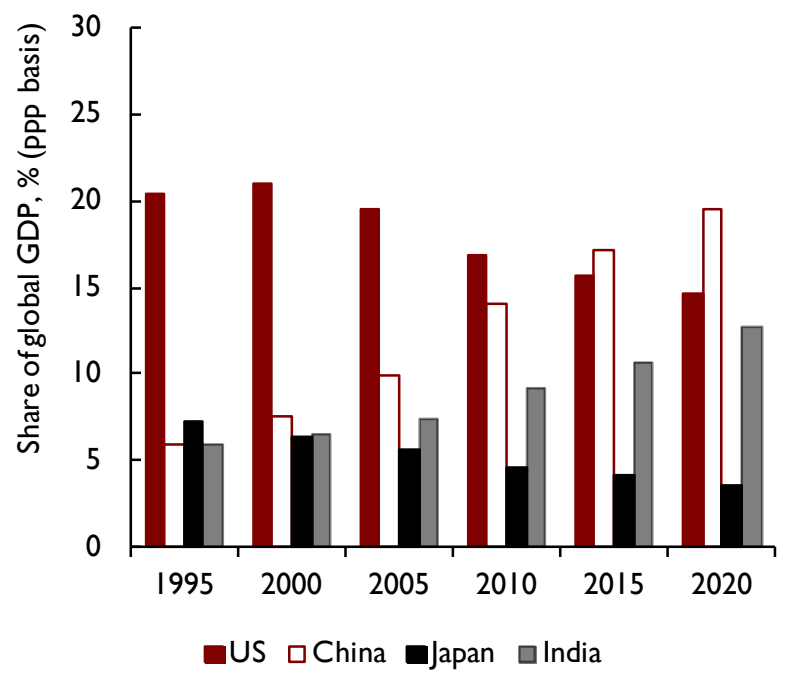


Table B4. United States

Percentage change

\begin{tabular}{|c|c|c|c|c|c|c|c|}
\hline & 2014 & 2015 & 2016 & 2017 & 2018 & 2019 & $\begin{array}{c}\text { Average } \\
2020-24\end{array}$ \\
\hline GDP & 2.6 & 2.9 & 1.5 & 2.3 & 2.7 & 2.6 & 2.2 \\
\hline Consumption & 2.9 & 3.6 & 2.7 & 2.8 & 2.7 & 2.7 & 1.7 \\
\hline Investment : housing & 3.5 & 10.2 & 5.5 & 1.8 & 5.3 & 6.4 & 3.6 \\
\hline : business & 6.9 & 2.3 & -0.6 & 4.7 & 5.5 & 5.2 & 3.1 \\
\hline Government: consumption & -0.5 & 1.3 & 1.0 & 0.1 & 1.4 & 1.5 & 1.6 \\
\hline : investment & -1.4 & 1.6 & -0.2 & 0.1 & 1.7 & 1.2 & 1.6 \\
\hline Stockbuilding(a) & -0.1 & 0.2 & -0.4 & -0.1 & 0.0 & 0.0 & 0.0 \\
\hline Total domestic demand & 2.7 & 3.5 & 1.6 & 2.4 & 3.0 & 2.9 & 1.9 \\
\hline Export volumes & 4.3 & 0.4 & -0.3 & 3.4 & 4.9 & 4.2 & 3.8 \\
\hline Import volumes & 4.5 & 5.0 & 1.3 & 4.0 & 6.3 & 5.8 & 2.1 \\
\hline Average earnings & 2.6 & 2.8 & 1.1 & 1.6 & 2.9 & 3.0 & 3.1 \\
\hline Private consumption deflator & 1.5 & 0.3 & 1.2 & 1.7 & 2.3 & 2.1 & 2.2 \\
\hline RPDI & 3.6 & 4.1 & 1.4 & I.I & 2.3 & 2.5 & 1.4 \\
\hline Unemployment, \% & 6.2 & 5.3 & 4.9 & 4.3 & 4.1 & 4.1 & 4.8 \\
\hline General Govt. balance as \% of GDP & -4.9 & -4.3 & -5.0 & -3.6 & -5.4 & -5.3 & -3.9 \\
\hline General Govt. debt as \% of GDP(b) & 103.0 & 104.1 & 105.3 & 103.2 & 103.4 & 104.0 & 102.7 \\
\hline Current account as \% of GDP & -2.1 & -2.4 & -2.4 & -2.4 & -3.1 & -3.4 & -3.3 \\
\hline
\end{tabular}

Note: (a) Change as a percentage of GDP. (b) End-of-year basis.

\begin{tabular}{|c|c|c|c|c|c|c|c|}
\hline & 2014 & 2015 & 2016 & 2017 & 2018 & 2019 & $\begin{array}{c}\text { Average } \\
2020-24\end{array}$ \\
\hline GDP & 2.9 & 1.0 & 1.4 & 3.0 & 2.6 & 2.3 & 1.7 \\
\hline Consumption & 2.6 & 2.2 & 2.3 & 3.4 & 2.8 & 1.7 & 1.2 \\
\hline Investment : housing & 2.2 & 3.8 & 3.3 & 3.1 & 3.7 & 2.9 & 2.7 \\
\hline : business & 4.5 & -11.0 & -9.2 & 2.7 & 4.8 & 2.2 & 0.8 \\
\hline Government: consumption & 0.5 & 1.6 & 2.2 & 2.2 & 2.2 & 1.9 & 1.7 \\
\hline : investment & -3.4 & 0.3 & 5.1 & 3.8 & 5.2 & 2.8 & 1.9 \\
\hline Stockbuilding(a) & -0.4 & -0.2 & -0.2 & 0.7 & 0.0 & 0.0 & 0.0 \\
\hline Total domestic demand & 1.8 & 0.3 & 1.0 & 3.8 & 3.0 & 1.9 & 1.4 \\
\hline Export volumes & 5.9 & 3.5 & 1.0 & 1.0 & 3.7 & 6.3 & 3.1 \\
\hline Import volumes & 2.3 & 0.7 & -1.0 & 3.6 & 4.8 & 4.8 & 2.2 \\
\hline Average earnings & 3.2 & 1.9 & 1.0 & 2.2 & 3.0 & 3.1 & 3.5 \\
\hline Private consumption deflator & 1.9 & I.I & 0.9 & I.I & 2.3 & 2.3 & 2.1 \\
\hline RPDI & 1.3 & 3.4 & 1.5 & 3.6 & 2.4 & 1.6 & 1.2 \\
\hline Unemployment, \% & 6.9 & 6.9 & 7.0 & 6.3 & 5.8 & 5.8 & 6.0 \\
\hline General Govt. balance as \% of GDP & 0.2 & -0.1 & -1.1 & -1.0 & -1.0 & -1.0 & -1.4 \\
\hline General Govt. debt as \% of GD(b)P & 91.1 & 97.8 & 96.6 & 95.6 & 91.6 & 88.2 & 82.7 \\
\hline Current account as $\%$ of GDP & -2.4 & -3.6 & -3.2 & -3.0 & -2.5 & -1.8 & -0.9 \\
\hline
\end{tabular}

Note: (a) Change as a percentage of GDP. (b) End-of-year basis. 
Table B6. Japan

Percentage change

\begin{tabular}{|c|c|c|c|c|c|c|c|}
\hline & 2014 & 2015 & 2016 & 2017 & 2018 & 2019 & $\begin{array}{c}\text { Average } \\
2020-24\end{array}$ \\
\hline GDP & 0.3 & 1.4 & 0.9 & 1.7 & 1.2 & 0.9 & 0.9 \\
\hline Consumption & -0.9 & 0.0 & 0.1 & 1.0 & 0.5 & 0.4 & I.I \\
\hline Investment: housing & -4.0 & -1.2 & 5.6 & 2.7 & -0.7 & 1.4 & 3.6 \\
\hline : business & 5.2 & 3.4 & 0.6 & 3.0 & 3.3 & 1.3 & 1.6 \\
\hline Government: consumption & 0.5 & 1.5 & 1.3 & 0.1 & 0.0 & 0.0 & 0.3 \\
\hline : investment & 0.6 & -1.3 & 0.1 & 1.3 & -0.1 & 0.2 & 0.3 \\
\hline Stockbuilding(a) & 0.1 & 0.3 & -0.2 & -0.1 & 0.3 & 0.0 & 0.0 \\
\hline Total domestic demand & 0.3 & 1.0 & 0.4 & 1.2 & 1.0 & 0.5 & I.I \\
\hline Export volumes & 9.3 & 3.0 & 1.3 & 6.8 & 5.9 & 4.4 & 3.4 \\
\hline Import volumes & 8.2 & 0.7 & -1.9 & 3.6 & 5.0 & 2.2 & 4.0 \\
\hline Average earnings & 0.9 & 0.9 & 1.7 & 0.9 & 1.3 & 2.3 & 2.0 \\
\hline Private consumption deflator & 2.0 & 0.4 & -0.5 & 0.2 & 0.8 & 1.3 & 1.3 \\
\hline RPDI & -1.7 & 0.8 & 2.3 & 1.6 & 0.1 & 0.6 & 1.3 \\
\hline Unemployment, \% & 3.6 & 3.4 & 3.1 & 2.8 & 2.4 & 3.0 & 3.3 \\
\hline Govt. balance as \% of GDP & -5.4 & -3.5 & -4.6 & -4.8 & -4.2 & -4.0 & -3.9 \\
\hline Govt. debt as \% of GDP(b) & 213.0 & 213.9 & 216.8 & 218.2 & 220.9 & 219.6 & $2 \mid 4.1$ \\
\hline Current account as $\%$ of GDP & 0.8 & 3.0 & 3.7 & 4.0 & 3.5 & 3.7 & 4.8 \\
\hline
\end{tabular}

Note: (a) Change as a percentage of GDP. (b) End-of-year basis.

Table B7. Euro Area

Percentage change

\begin{tabular}{|c|c|c|c|c|c|c|c|}
\hline & 2014 & 2015 & 2016 & 2017 & 2018 & 2019 & $\begin{array}{c}\text { Average } \\
2020-24\end{array}$ \\
\hline GDP & 1.4 & 2.0 & 1.8 & 2.5 & 2.3 & 1.9 & 1.4 \\
\hline Consumption & 0.9 & 1.8 & 1.9 & 1.7 & 1.8 & 1.7 & 1.2 \\
\hline Private investment & 2.3 & 3.2 & 3.4 & 4.7 & 4.6 & 3.5 & 2.0 \\
\hline Government : consumption & 0.7 & 1.3 & 1.8 & 1.2 & 1.5 & 1.4 & 1.3 \\
\hline : investment & -0.7 & 2.9 & 1.1 & 1.7 & 2.6 & 2.6 & 1.1 \\
\hline Stockbuilding(a) & 0.3 & 0.0 & -0.1 & 0.0 & 0.0 & 0.0 & 0.0 \\
\hline Total domestic demand & 1.3 & 2.0 & 2.0 & 2.1 & 2.2 & 2.0 & 1.4 \\
\hline Export volumes & 4.6 & 6.1 & 3.4 & 5.3 & 4.8 & 3.5 & 2.5 \\
\hline Import volumes & 4.9 & 6.5 & 4.8 & 4.3 & 4.9 & 4.0 & 2.7 \\
\hline Average earnings & 1.3 & 1.4 & 1.5 & 1.4 & 2.1 & 2.3 & 2.7 \\
\hline Harmonised consumer prices & 0.4 & 0.0 & 0.2 & 1.5 & 1.6 & 1.6 & 1.6 \\
\hline RPDI & 0.8 & 1.3 & 1.9 & 2.1 & 1.7 & 2.1 & 1.6 \\
\hline Unemployment, \% & 11.6 & 10.9 & 10.0 & 9.1 & 8.4 & 8.0 & 7.9 \\
\hline Govt. balance as \% of GDP & -2.6 & -2.1 & -1.5 & -0.7 & -0.5 & -0.5 & -1.4 \\
\hline Govt. debt as \% of GDP(b) & 92.5 & 90.6 & 89.6 & 86.9 & 83.6 & 80.8 & 75.4 \\
\hline Current account as \% of GDP & 2.4 & 3.2 & 3.4 & 3.5 & 3.5 & 3.4 & 3.4 \\
\hline
\end{tabular}

Note: (a) Change as a percentage of GDP. (b) End-of-year basis; Maastricht definition. 
Table B8. Germany

Percentage change

\begin{tabular}{|c|c|c|c|c|c|c|c|}
\hline & 2014 & 2015 & 2016 & 2017 & 2018 & 2019 & $\begin{array}{c}\text { Average } \\
2020-24\end{array}$ \\
\hline GDP & 1.9 & 1.5 & 1.9 & 2.5 & 2.4 & 1.9 & 1.2 \\
\hline Consumption & 1.0 & 1.6 & 1.9 & 2.1 & 2.4 & 2.1 & 0.6 \\
\hline Investment : housing & 3.1 & -1.2 & 3.8 & 3.6 & 1.6 & 2.0 & 1.7 \\
\hline : business & 4.8 & 1.4 & 2.5 & 4.0 & 4.3 & 3.2 & 1.4 \\
\hline Government: consumption & 1.5 & 2.9 & 3.7 & 1.6 & 1.8 & $\mathrm{I} .4$ & 0.8 \\
\hline : investment & -1.2 & 4.5 & 2.6 & 4.6 & 5.0 & 6.5 & 0.1 \\
\hline Stockbuilding(a) & -0.3 & -0.3 & -0.1 & 0.0 & 0.2 & 0.0 & 0.0 \\
\hline Total domestic demand & 1.3 & 1.5 & 2.4 & 2.4 & 2.8 & 2.2 & 0.8 \\
\hline Export volumes & 4.5 & 4.7 & 2.4 & 5.3 & 5.3 & 4.2 & 2.7 \\
\hline Import volumes & 3.5 & 5.2 & 3.8 & 5.6 & 6.6 & 5.3 & 2.2 \\
\hline Average earnings & 2.5 & 3.0 & 2.9 & 2.5 & 3.0 & 2.5 & 2.6 \\
\hline Harmonised consumer prices & 0.8 & 0.1 & 0.4 & 1.7 & 1.7 & 1.7 & 1.6 \\
\hline RPDI & 1.5 & 1.9 & 2.1 & 2.4 & 1.3 & 1.7 & 0.8 \\
\hline Unemployment, \% & 5.0 & 4.7 & 4.2 & 3.8 & 3.6 & 3.6 & 4.0 \\
\hline Govt. balance as \% of GDP & 0.3 & 0.6 & 0.8 & 1.1 & 0.9 & 0.5 & -0.6 \\
\hline Govt. debt as \% of GDP(b) & 74.7 & 71.0 & 68.2 & 64.1 & 59.6 & 56.2 & 49.9 \\
\hline Current account as $\%$ of GDP & 7.5 & 9.0 & 8.5 & 8.1 & 7.9 & 7.5 & 7.4 \\
\hline
\end{tabular}

Note: (a) Change as a percentage of GDP. (b) End-of-year basis; Maastricht definition.

Table B9. France

\begin{tabular}{|c|c|c|c|c|c|c|c|}
\hline & 2014 & 2015 & 2016 & 2017 & 2018 & 2019 & $\begin{array}{c}\text { Average } \\
2020-24\end{array}$ \\
\hline GDP & 1.0 & 1.0 & $\mathrm{I} . \mathrm{I}$ & 2.0 & 1.9 & 1.9 & 1.5 \\
\hline Consumption & 0.7 & 1.3 & 2.1 & 1.3 & $\mathrm{I} .4$ & 1.8 & 1.5 \\
\hline Investment : housing & -3.0 & -2.1 & 2.4 & 5.3 & 3.4 & 5.2 & 6.5 \\
\hline : business & 2.9 & 3.1 & 3.6 & 4.4 & 4.5 & 3.6 & 2.0 \\
\hline Government: consumption & 1.3 & 1.1 & 1.2 & 1.6 & 1.4 & 1.2 & 1.7 \\
\hline : investment & -5.4 & -3.0 & -0.1 & -1.0 & 1.8 & 1.6 & 1.8 \\
\hline Stockbuilding(a) & 0.7 & 0.3 & -0.1 & 0.4 & -0.3 & 0.0 & 0.0 \\
\hline Total domestic demand & 1.5 & 1.5 & 1.9 & 2.3 & 1.6 & 2.0 & 1.9 \\
\hline Export volumes & 3.4 & 4.0 & 1.9 & 3.3 & 5.2 & 4.1 & 2.4 \\
\hline Import volumes & 4.8 & 5.5 & 4.2 & 4.1 & 3.7 & 4.3 & 3.3 \\
\hline Average earnings & 1.5 & 0.3 & 1.6 & 2.1 & 1.9 & 1.8 & 3.1 \\
\hline Harmonised consumer prices & 0.6 & 0.1 & 0.3 & 1.2 & 1.6 & 1.4 & 1.7 \\
\hline RPDI & 0.7 & 1.2 & 2.0 & 1.6 & 1.6 & 1.9 & 2.0 \\
\hline Unemployment, \% & 10.3 & 10.4 & 10.1 & 9.4 & 8.9 & 8.4 & 7.3 \\
\hline Govt. balance as $\%$ of GDP & -3.9 & -3.6 & -3.4 & -2.6 & -1.9 & -1.7 & -2.2 \\
\hline Govt. debt as \% of GDP(b) & 94.9 & 95.8 & 96.6 & 96.7 & 95.2 & 93.7 & 89.7 \\
\hline Current account as $\%$ of GDP & -1.3 & -0.4 & -0.9 & -1.2 & -0.8 & -0.5 & -1.0 \\
\hline
\end{tabular}

Note: (a) Change as a percentage of GDP. (b) End-of-year basis; Maastricht definition. 
Table BI0. Italy

Percentage change

\begin{tabular}{|c|c|c|c|c|c|c|c|}
\hline & 2014 & 2015 & 2016 & 2017 & 2018 & 2019 & $\begin{array}{c}\text { Average } \\
2020-24\end{array}$ \\
\hline GDP & 0.2 & 0.8 & 1.0 & 1.5 & 1.4 & 1.3 & 1.2 \\
\hline Consumption & 0.2 & 1.9 & 1.4 & 1.3 & 0.9 & 0.6 & 0.7 \\
\hline Investment : housing & -6.8 & -1.7 & 2.9 & 2.2 & 2.9 & 2.0 & 1.7 \\
\hline : business & 0.6 & 4.0 & 4.3 & 5.6 & 7.2 & 2.1 & 1.7 \\
\hline Government : consumption & -0.7 & -0.6 & 0.6 & 0.1 & 0.9 & I.I & 0.9 \\
\hline : investment & -5.4 & -1.2 & -1.0 & -2.5 & 2.9 & 2.8 & 1.0 \\
\hline Stockbuilding(a) & 0.7 & 0.0 & -0.3 & -0.2 & -0.4 & 0.0 & 0.0 \\
\hline Total domestic demand & 0.3 & 1.4 & 1.3 & 1.3 & 1.3 & 1.0 & 0.9 \\
\hline Export volumes & 2.4 & 4.2 & 2.6 & 6.0 & 3.9 & 2.7 & 2.4 \\
\hline Import volumes & 3.0 & 6.6 & 3.8 & 5.7 & 4.2 & 1.7 & 1.7 \\
\hline Average earnings & 0.4 & 0.6 & 0.2 & -0.4 & 1.4 & 2.5 & 1.9 \\
\hline Harmonised consumer prices & 0.2 & 0.1 & -0.1 & 1.3 & 1.3 & 1.5 & 1.4 \\
\hline RPDI & 0.5 & 0.5 & 1.4 & 1.2 & 1.8 & 2.3 & 1.0 \\
\hline Unemployment, \% & 12.6 & 11.9 & 11.7 & 11.3 & 10.8 & 10.5 & 10.5 \\
\hline Govt. balance as \% of GDP & -3.0 & -2.6 & -2.5 & -1.6 & -1.1 & -1.0 & -1.9 \\
\hline Govt. debt as \% of GDP(b) & 131.7 & 131.6 & 131.9 & 132.1 & 128.2 & 124.7 & 118.7 \\
\hline Current account as $\%$ of GDP & 1.9 & 1.5 & 2.7 & 3.0 & 3.1 & 3.9 & 4.9 \\
\hline
\end{tabular}

Note: (a) Change as a percentage of GDP. (b) End-of-year basis; Maastricht definition.

Table BI I. Spain

Percentage change

\begin{tabular}{|c|c|c|c|c|c|c|c|}
\hline & 2014 & 2015 & 2016 & 2017 & 2018 & 2019 & $\begin{array}{c}\text { Average } \\
2020-24\end{array}$ \\
\hline GDP & 1.4 & 3.4 & 3.3 & 3.1 & 2.5 & 2.0 & 1.7 \\
\hline Consumption & 1.5 & 3.0 & 3.0 & 2.4 & 2.3 & 1.8 & 1.4 \\
\hline Investment : housing & 11.3 & -1.0 & 4.4 & 8.3 & 4.6 & 3.0 & 3.8 \\
\hline : business & -2.5 & 7.7 & 3.2 & 3.8 & 4.5 & 2.8 & 2.8 \\
\hline Government: consumption & -0.3 & 2.1 & 0.8 & 1.6 & 1.4 & 1.8 & 1.9 \\
\hline : investment & 8.8 & 16.5 & 2.2 & 2.5 & -0.1 & 1.8 & 1.9 \\
\hline Stockbuilding(a) & 0.2 & 0.4 & 0.0 & 0.1 & 0.1 & 0.0 & 0.0 \\
\hline Total domestic demand & 2.0 & 4.0 & 2.6 & 2.9 & 2.4 & 2.0 & 1.9 \\
\hline Export volumes & 4.3 & 4.2 & 4.8 & 5.0 & 3.3 & 3.8 & 2.6 \\
\hline Import volumes & 6.6 & 5.9 & 2.7 & 4.7 & 3.1 & 3.9 & 3.3 \\
\hline Average earnings & -0.1 & 1.9 & 0.4 & 0.7 & I.I & 2.0 & 2.9 \\
\hline Harmonised consumer prices & -0.2 & -0.6 & -0.3 & 2.0 & 1.7 & 1.5 & 1.6 \\
\hline RPDI & 1.2 & 2.3 & 1.9 & 1.5 & 2.1 & 2.4 & 1.7 \\
\hline Unemployment, \% & 24.5 & 22.1 & 19.6 & 17.2 & 15.0 & 13.2 & 13.1 \\
\hline Govt. balance as \% of GDP & -6.0 & -5.3 & -4.5 & -3.3 & -2.1 & -1.8 & -2.2 \\
\hline Govt. debt as $\%$ of GDP(b) & 100.4 & 99.4 & 99.0 & 97.5 & 94.7 & 92.4 & 87.9 \\
\hline Current account as \% of GDP & 1.0 & $\mathrm{I} . \mathrm{I}$ & 1.9 & 2.0 & 1.7 & 2.3 & 2.1 \\
\hline
\end{tabular}

Note: (a) Change as a percentage of GDP. (b) End-of-year basis; Maastricht definition. 